\title{
Comment on: Prevalence of growth and endocrine disorders in Malaysian children with transfusion-dependent thalassaemia
}

Singapore Med J 2019; 60(12): 657 https://doi.org/10.11622/smedj.2019163

Dear Sir,

We read the article "Prevalence of growth and endocrine disorders in Malaysian children with transfusion-dependent thalassaemia" with great interest. ${ }^{(1)}$ Tan et al concluded that "Thyroid dysfunction and hypoparathyroidism may be missed if endocrine screening is only performed in children with TDT [transfusion-dependent thalassaemia] > 10 years of age. Close monitoring for endocrine dysfunction and hormonal therapy is essential to prevent long-term adverse outcomes.". ${ }^{(1)}$

We would like to share our thoughts on this observation. Thalassaemia is highly prevalent in our setting, Thailand. The incidence of hypothyroidism is also very high, and prenatal screening for thalassaemia and neonatal screening for hypothyroidism are presently practised in Thailand. ${ }^{(2)}$ However, the relationship between thalassaemia disorder and thyroid disorder may be a coincidence due to the high incidence of both diseases. Regarding the adverse effect of polytransfusion in children with TDT, disturbance of the endocrine system might be possible but requires a long period of exposure to the blood product. In a case with chronic anaemia due to thalassaemia, Soliman et al found that the incidence of thyroid function impairment was related to "increasing prevalence with age, severity of the anaemia and higher ferritin concentration", which is a result of polytransfusion. (3) If a practitioner is looking for endocrine disruption that is a direct complication of polytransfusion, the problem might not be detectable early.

Yours sincerely,

Pathum $\underline{\text { Sookaromdee }}{ }^{1}$, Viroj $\underline{\text { Wiwanitkit }}^{2}$

${ }^{1}$ TWS Medical Center, Bangkok, Thailand, 2Dr DY Patil University, Pune, India. pathumsook@gmail.com

\section{References}

1. Tan KA, Lum SH, Yahya A, et al. Prevalence of growth and endocrine disorders in Malaysian children with transfusion-dependent thalassaemia. Singapore Med J 2019; 60:303-8

2. Limwongse C. Medical genetic services in a developing country: lesson from Thailand. Curr Opin Pediatr 2017; 29:634-9.

3. Soliman AT, De Sanctis V, Yassin M, Wagdy M, Soliman N. Chronic anemia and thyroid function. Acta Biomed 2017; 88:119-27. 